active mucocutaneous pemphigus vulgaris (20). Inhibition ELISAs showed that epitopes defined by these monoclonal antibodies are blocked by autoantibodies from multiple patients with pemphigus. Moreover, genetic analysis of these antibodies revealed restricted patterns of heavy- and light-chain gene utilization. Taken together, these findings suggest several novel approaches to abrogating disease (e.g., targeting $B$ cells that express certain $V_{H}$ gene segments, generating peptides that may block pathogenic antibodies reactive with shared and dominant epitopes, or using blocking anti-idiotypic antibodies). These approaches, coupled with better understanding of the contributions of molecular mimicry as well as mechanisms of central and peripheral tolerance to disease pathogenesis, will facilitate the development of effective interventions that counteract the chronic morbidity (i.e., pruritus, blistering, infection, scarring, and treatment-related immunosuppression) and, at times, mortality of immunobullous diseases.

\section{Acknowledgments}

This work was supported in part by a grant from the NIH (RO1 AR048982-01A1) to the author.
Address correspondence to: Kim B. Yancey, Medical College of Wisconsin, Department of Dermatology, 8701 Watertown Plank Road, Milwaukee, Wisconsin 53226-4801, USA. Phone: (414) 456-4081; Fax: (414) 456-6518; E-mail: kyancey@mcw.edu.

1. Udey, M.C., and Stanley, J.R. 1999. Pemphigus-diseases of antidesmosomal autoimmunity. JAMA. 282:572-576

2. Yancey, K.B., and Egan, C.A. 2000. Pemphigoid: clinical, histologic, immunopathologic, and therapeutic considerations. JAMA. 284:350-356.

3. Schmidt, E., and Zillikens, D. 2000. Autoimmune and inherited subepidermal blistering diseases: advances in the clinic and the laboratory. Adv. Dermatol. 16:113-157.

4. Burgeson, R.E., and Christiano, A.M. 1997. The dermal-epidermal junction. Curr. Opin. Cell Biol. 9:651-658.

5. Liu, Z., et al. 2005. Synergy between a plasminogen cascade and MMP-9 in autoimmune disease. J. Clin. Invest. 115:879-887. doi:10.1172/JCI200523977.

6. Sitaru, C., et al. 2005. Induction of dermal-epidermal separation in mice by passive transfer of antibodies to type VII collagen. J. Clin. Invest. 115:870-878. doi:10.1172/JCI200521386.

7. Van den Bergh, F., and Giudice, G.J. 2003. BP180 (type XVII collagen) and its role in cutaneous biology and disease. Adv. Dermatol. 19:37-71.

8. Masunaga, T., et al. 1997. The extracellular domain of BPAG2 localizes to anchoring filaments and its carboxyl terminus extends to the lamina densa of normal human epidermal basement membrane. J. Invest. Dermatol. 109:200-206.

9. Nonaka, S., et al. 2000. The extracellular domain of BPAG2 has a loop structure in the carboxy terminal flexible tail in vivo. J. Invest. Dermatol. 115:889-892.

10. Giudice, G.J., et al. 1993. Bullous pemphigoid and herpes gestationis autoantibodies recognize a common non-collagenous site on the BP180 ectodomain. J. Immunol. 151:5742-5750.

11. Liu, Z., et al. 1993. A passive transfer model of the organ-specific autoimmune disease, bullous pemphigoid, using antibodies generated against the hemidesmosomal antigen, BP180. J. Clin. Invest. 92:2480-2488

12. Liu, Z., et al. 1995. The role of complement in experimental bullous pemphigoid. J. Clin. Invest. 95:1539-1544.

13. Liu, Z., et al. 1997. A major role for neutrophils in experimental bullous pemphigoid. J. Clin. Invest. 100:1256-1263.

14. Liu, Z., et al. 1998. Gelatinase B-deficient mice are resistant to experimental bullous pemphigoid. J. Exp. Med. 188:475-482.

15. Liu, Z., et al. 2000. A critical role for neutrophil elastase in experimental bullous pemphigoid. J. Clin. Invest. 105:113-123.

16. Liu, Z., et al. 2000. The serpin alpha1-proteinase inhibitor is a critical substrate for gelatinase B/ MMP-9 in vivo. Cell. 102:647-655.

17. Woodley, D.T., et al. 1984. Identification of the skin basement-membrane autoantigen in epidermolysis bullosa acquisita. N. Engl. J. Med. 310:1007-1013.

18. Woodley, D.T., et al. 1988. Epidermolysis bullosa acquisita antigen is the globular carboxyl terminus of type VII procollagen. J. Clin. Invest. 81:683-687.

19. Shimizu, H., et al. 1997. Most anchoring fibrils in human skin originate and terminate in the lamina densa. Lab. Invest. 76:753-763.

20. Payne, A.S., et al. 2005. Genetic and functional characterization of human pemphigus vulgaris monoclonal autoantibodies isolated by phage display. J. Clin. Invest. 115:888-899. doi:10.1172/ JCI200524185.

\title{
To live or die: a critical decision for the lung
}

\author{
G.R. Scott Budinger and Jacob I. Sznajder
}

Division of Pulmonary and Critical Care Medicine, Northwestern University, Chicago, Illinois, USA.

\begin{abstract}
Every cell in the body expresses a set of proteins designed to trigger permeabilization of the mitochondria and cell death. Inactivation or inappropriate triggering of these pathways is increasingly recognized as a contributor to human disease. A study in this issue of the JCI demonstrates that IL-6 exerts its protective effect against the development of lung injury following exposure of mice to $95 \% \mathrm{O}_{2}$ by increasing the expression of a Bcl-2-related protein, $\mathrm{A1}$ (see the related article beginning on page 1039). This protein acts to prevent mitochondrial membrane permeabilization and cell death following exposure to hyperoxia. The data in this study lend support to the hypothesis that inappropriate triggering of cell-death pathways may contribute to the development of hyperoxic pulmonary edema, lung injury, and respiratory failure.
\end{abstract}

To maintain homeostasis, cell growth must be precisely balanced by cell death. It is there-

Nonstandard abbreviations used: $\mathrm{BH}, \mathrm{Bcl}-2$ homology; KGF, keratinocyte growth factor.

Conflict of interest: The authors have declared that no conflict of interest exists.

Citation for this article: J. Clin. Invest. 115:828-830 (2005). doi:10.1172/JCI200524681. fore not surprising that long-lived mammals have highly conserved, complex pathways that regulate cell death. The importance of these pathways has been demonstrated during embryogenesis and in many pathologic conditions (1). Disturbance of the cell-death pathways can result in either malignancy or autoimmune disorders, while premature cell death has been implicated in the devel- opment of degenerative diseases, immunodeficiency, and infertility (1). Studies of the apoptotic pathways in the past decade have led to the realization that, at a molecular level, cells in the body are poised to "sacrifice" themselves for the benefit of the organism. This has led some investigators to speculate that inappropriate activation of these pathways may explain the convergence of a number of different injurious stimuli in the phenotypes of either lung injury or multiple organ dysfunction syndrome (2).

In many nonimmune mammalian cells, death pathways converge at the mitochondria, which serve a dual role as the primary metabolic engines of the cell and as critical cell-death checkpoints. As depicted schematically in Figure 1, exposure to a death signal results in the permeabilization of the mitochondrial outer membrane, releasing a variety of proteins, including cytochrome $c$, 


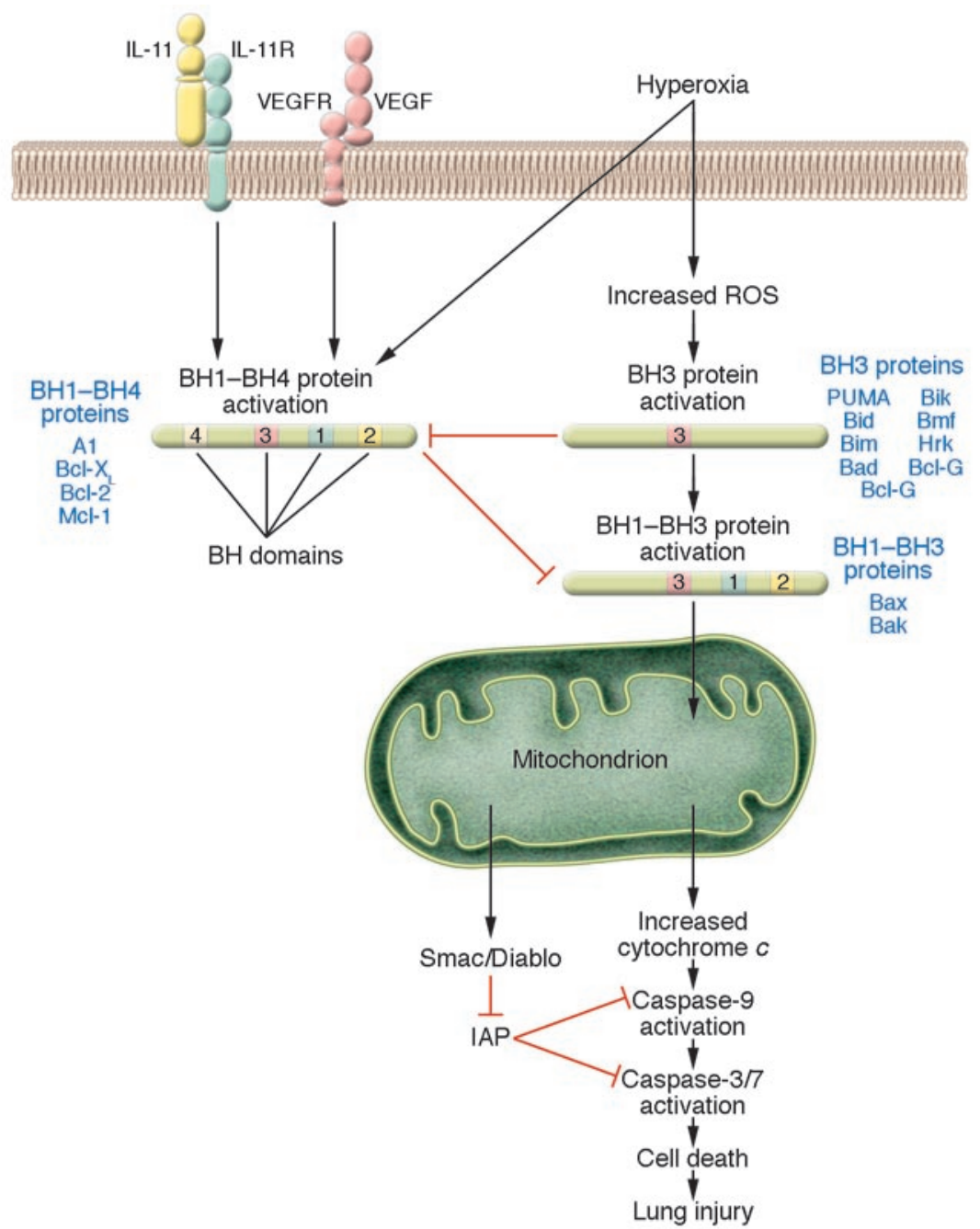

Smac/Diablo, and other proapoptotic molecules that result in cell death. Proteins of the Bcl-2 family regulate mitochondrial membrane permeabilization during cell death. These proteins share up to $4 \mathrm{Bcl}-2$ homology $(\mathrm{BH})$ domains, which are required for protein function. Proteins that contain $3 \mathrm{BH}$ domains (BH1-BH3), for example Bax and Bak, can oligomerize to form channels in the outer mitochondrial membrane sufficiently large to release cytochrome $c$ and other macromolecules. These proteins are held in check by proteins sharing $4 \mathrm{BH}$ domains (BH1-BH4), for example Bcl-2, Bcl- $\mathrm{X}_{\mathrm{L}}$, and A1 (1). Proteins containing a single $\mathrm{BH}$ domain ( $\mathrm{BH} 3$ proteins) initiate apoptosis by activating Bax or Bak or by inhibiting $\mathrm{BH} 1-\mathrm{BH} 4$ proteins.

\section{Hyperoxia-induced lung injury is regulated by signaling events}

Exposure to hyperoxia is a well-established model of lung injury characterized by the development of pulmonary edema and inflammation (3). The development of hyperoxic lung injury has been clearly shown to require the generation of reactive oxygen species, which leads to alveolar epithelial and endothelial cell death by both apoptosis and necrosis (4). Overexpression of cytokines or chemokines (e.g., TNF- $\alpha$, IL-1 $\beta$, IL-6, CXC-chemokine receptor 2, and IL-11), growth factors (e.g., IGF and keratinocyte growth factor [KGF]), Akt, or the $\beta$ subunit of the Na,K-ATPase has been shown to protect animals from hyperoxia, which suggests that hyperoxic lung injury results from the activation of cellular-signaling pathways that are susceptible to modulation (5-8). The mechanisms by which these events protect against injury, however, have not been completely elucidated. In this issue of the JCI, He et al. (9) describe new findings based on the previous observation that mice overexpressing IL-6 were protected against hyperoxia-

\section{Figure 1}

Schematic representation of the cell-death pathways activated by exposure to hyperoxia. The $\mathrm{Bcl}-2$ family of proteins is a group that contains $1 \mathrm{BH}$ domain $(\mathrm{BH} 3)$ or $3 \mathrm{BH}$ domains ( $\mathrm{BH} 1-\mathrm{BH} 3)$, both of which induce cell death, and also contains $4 \mathrm{BH}$ domains (BH1-BH4), which protect against cell death (see text for details). In this issue of the $\mathrm{JCl}, \mathrm{He}$ et al. (9) report that IL-11 induces expression of the antiapoptotic Bcl-2related protein $\mathrm{A} 1$, which is required for IL-11induced protection against hyperoxiainduced lung injury. IAP, inhibitor of apoptosis protein; IL-11R, IL-11 receptor.

induced lung injury through a mechanism that was independent of the expression of antioxidant enzymes (10). Building upon other investigators' work implicating involvement of the Bcl-2 family of proteins in hyperoxia-induced lung injury $(11,12)$, $\mathrm{He}$ and colleagues sought to determine whether regulation of these proteins by IL-11 might explain its protective effect. They found that IL-11 upregulated the expression of an antiapoptotic Bcl-2related protein, A1. This protein and its human counterpart, Bfl-1, contain $4 \mathrm{BH}$ domains but lack a C-terminal transmembrane domain found in many other Bcl-2 proteins (13). Bfl-1/A1 is expressed in the immune cells, the spleen, and the lung, and functionally acts to prevent cell death in a manner similar to that of $\mathrm{Bcl}-2$ and $\mathrm{Bcl}-\mathrm{X}_{\mathrm{L}}$ (13-16). Mice lacking A1 showed increased susceptibility to hyperoxia-induced lung injury, and overexpression of IL-11 was no longer protective in the A1-knockout animals (9). These in vivo findings were confirmed in a mouse lung epithelial cell line, in which the overexpression of A1 protected against hyperoxia-induced necrosis and apoptosis.

\section{Cell death and lung injury}

In this important report, $\mathrm{He}$ et al. have demonstrated a clear role for the Bcl-2 family of proteins in the development of hyperoxic lung injury (9). As the only role yet identified for the Bcl-2 family of proteins is the regulation of cell death, these new data suggest that alveolar epithelial and/or endothelial cell death is important in the pathophysiology of lung injury. If so, this finding could provide a potential explanation for the protection conferred by seemingly disparate stimuli against hyperoxic lung injury. For example, treatment with TNF- $\alpha$ or IL- $1 \beta$ might stimulate 
$\mathrm{NF}-\kappa \mathrm{B}-$ dependent transcription of antiapoptotic Bcl-2 family members. Also, growth factors such as KGF might prevent hyperoxia-induced cell death through the activation of Akt (17). The overexpression of Akt has been shown to prevent mitochondria-dependent apoptosis independently of the $\mathrm{Bcl}-2$ proteins in vitro and to prevent hyperoxia-induced lung injury in vivo $(7,18)$.

\section{Implications for future research}

Establishing a role for the Bcl-2 family of proteins in hyperoxic lung injury will likely influence future investigations into the molecular mechanisms by which these pathways become activated to contribute to hyperoxic and other forms of acute lung injury. For example, the link between the intracellular or extracellular generation of oxidants and the activation of the Bcl-2 family of proteins is not known. Additionally, the most established mechanism by which $\mathrm{Bcl}-2$-related proteins induce cell death is through permeabilization of the outer membrane of the mitochondria; however, these proteins can also form channels in the ER that result in the release of ER calcium and cell death $(19,20)$. Hyperoxia, like other forms of oxidant stress, might also induce cell death through this ERdependent mechanism.

Many investigators, including $\mathrm{He}$ et al., have observed that the morphology of cell death, in vitro and in vivo, is both necrotic and apoptotic, which raises the question of how activation of the mitochondria-dependent apoptotic pathway might result in both of these phenotypes. Mitochondrial membrane permeabilization by $\mathrm{Bcl}-2$ proteins results in the activation of caspases, cysteine proteases that use energy derived from ATP hydrolysis to cleave their substrates after an aspartic acid residue. Activation of these caspases is required for cells to die by apoptosis, yet caspase activation has been more difficult to detect following exposure to hyperoxia than in other forms of apoptotic cell death (5). Is this the result of activation of caspase-independent cell-death pathways that are also regulated by the Bcl-2 family of proteins, or do derangements in cell metabolism induced by hyperoxia prevent hyperoxia-induced caspase activation?

Finally, not all strategies shown to prevent hyperoxia-induced lung injury have an obvious link to cell death. For example, overexpression of Na,K-ATPase, which restores alveolar epithelial cell polarity and increases lung edema clearance, protects against hyperoxic lung injury (6). Do these approaches increase the resistance of the cells to death stimuli, or do they act through an independent mechanism to prevent lung injury? The work by He et al. (9) brings into focus novel lines of investigation aimed at dissecting those pathways of cell death that have significant relevance to acute lung injury.

\section{Acknowledgments}

This work was supported in part by NIH grants HL-48129, K08HL-67835, and PO1 HL-71643.

Address correspondence to: Jacob I. Sznajder, Northwestern University, 240 E. Huron, McGaw 2nd Floor, Chicago, Illinois 60611, USA. Phone: (312) 908-8163; Fax: (312) 9084650; E-mail: j-sznajder@northwestern.edu.

1. Danial, N.N., and Korsmeyer, S.J. 2004. Cell death: critical control points. Cell. 116:205-219.

2. Martin, T.R., Nakamura, M., and Matute-Bello, G. 2003. The role of apoptosis in acute lung injury. Crit. Care. Med. 31:S184-S188.

3. Crapo, J.D., Barry, B.E., Foscue, H.A., and Shelburne, J. 1980. Structural and biochemical changes in rat lungs occurring during exposures to lethal and adaptive doses of oxygen. Am. Rev. Respir. Dis. 122:123-143.

4. Freeman, B.A., and Crapo, J.D. 1981. Hyperoxia increases oxygen radical production in rat lungs and lung mitochondria. J. Biol. Chem. 256:10986-10992.

5. Barazzone, C., and White, C.W. 2000. Mecha- nisms of cell injury and death in hyperoxia: role of cytokines and Bcl-2 family proteins. Am. J. Respir. Cell Mol. Biol. 22:517-519.

6. Factor, P., Dumasius, V., Saldias, F., Brown, L.A., and Sznajder, J.I. 2000. Adenovirus-mediated transfer of an $\mathrm{Na}+/ \mathrm{K}+$-ATPase beta 1 subunit gene improves alveolar fluid clearance and survival in hyperoxic rats. Hum. Gene Ther. 11:2231-2242.

7. Lu, Y., et al. 2001. Activated Akt protects the lung from oxidant-induced injury and delays death of mice. J. Exp. Med. 193:545-549.

8. Sue, R.D., et al. 2004. CXCR2 is critical to hyperoxiainduced lung injury. J. Immunol. 172:3860-3868.

9. He, C.H., et al. 2005. Bcl-2-related protein A1 is an endogenous and cytokine-stimulated mediator of cytoprotection in hyperoxic acute lung injury. J. Clin. Invest. 115:1039-1048. doi:10.1172/ JCI200523004.

10. Ward, N.S., et al. 2000. Interleukin-6-induced protection in hyperoxic acute lung injury. Am. J. Respir. Cell Mol. Biol. 22:535-542.

11. Wang, X., et al. 2003. Necrotic cell death in response to oxidant stress involves the activation of the apoptogenic caspase-8/bid pathway. J. Biol. Chem. 278:29184-29191.

12. Buccellato, L.J., Tso, M., Akinci, O.I., Chandel, N.S., and Budinger, G.R. 2004. Reactive oxygen species are required for hyperoxia-induced Bax activation and cell death in alveolar epithelial cells. J. Biol. Chem. 279:6753-6760.

13. Zhang, H., et al. 2000. Structural basis of BFL-1 for its interaction with BAX and its anti-apoptotic action in mammalian and yeast cells. J. Biol. Chem. 275:11092-11099.

14. Choi, S.S., et al. 1995. A novel Bcl-2 related gene, Bfl-1, is overexpressed in stomach cancer and preferentially expressed in bone marrow. Oncogene. 11:1693-1698.

15. Choi, S.S., Park, S.H., Kim, U.J., and Shin, H.S. 1997. Bfl-1, a Bcl-2-related gene, is the human homolog of the murine $\mathrm{A} 1$, and maps to chromosome 15q24.3. Mamm. Genome. 8:781-782.

16. D'Sa-Eipper, C., and Chinnadurai, G. 1998. Functional dissection of Bfl-1, a Bcl-2 homolog: antiapoptosis, oncogene-cooperation and cell proliferation activities. Oncogene. 16:3105-3114.

17. Pan, Z.Z., Devaux, Y., and Ray, P. 2004. Ribosomal S6 kinase as a mediator of keratinocyte growth factor-induced activation of Akt in epithelial cells. Mol. Biol. Cell. 15:3106-3113.

18. Majewski, N., et al. 2004. Hexokinase-mitochondria interaction mediated by Akt is required to inhibit apoptosis in the presence or absence of Bax and Bak. Mol. Cell. 16:819-830.

19. Zong, W.X., et al. 2003. Bax and Bak can localize to the endoplasmic reticulum to initiate apoptosis. J. Cell. Biol. 162:59-69.

20. Scorrano, L., et al. 2003. BAX and BAK regulation of endoplasmic reticulum $\mathrm{Ca} 2+$ : a control point for apoptosis. Science. 300:135-139. 